\title{
Eva Weißmann: Lernen im Gleichgewicht. Wie Bewegung die emotionale und kognitive Entwicklung fördert
}

\author{
Brandes \& Apsel, 2016, Frankfurt/M., 268 Seiten, 24,90€
}

n einer umfangreichen, interdisziplinären Veröffentlichung legt Eva Weißmann den Zusammenhang von Bewegung/Körper, Psyche und Kognition im Lernprozess dar. Dabei schlägt sie einen Bogen von ihrem Bewegungswissen als Tänzerin und Feldenkrais-Lehrerin zur interpersonellen Neurobiologie von D. Siegel, zur Psychologie, speziell Winnicott, und zur Säuglings- und Bindungsforschung von Stern.

Ein komplexes Thema wie Lernentwicklung, in der sich die Faktoren reziprok beeinflussen und schließlich in neuronalen Prägungen ihre Entsprechung finden, lässt sich schwer linear darstellen und hat auch keinen eindeutigen Beginn. Weißmann löst das Dilemma, indem sie die Leserlnnen einlädt, selbst zu entscheiden, von welchem Kapitel bzw. Sichtweise aus er / sie das Thema aufrollen möchte, und findet damit gleich eine Form, ihren eigenen Thesen zu entsprechen: Da wo das eigene Interesse liegt, ist auch die größte Aufmerksamkeit aktiviert, und Lernwege sind immer individuell.

Manche Kapitel umfassen praktische Ansätze: frühes Infant-Handling, Auszüge aus der Feldenkrais-Methode und Übungen aus dervon der Autorin speziell entwickelten „Kinästhetisch orientierten Pädagogik (KOP)“. Alle Kapitel stellen die Bedeutung von aufmerksamer Hinwendung und achtsamer Berührung, von Stimmklang/Prosodie im Kontakt sowie von der Entwicklung der kindlichen kinästhetischen Kompetenz durch Bewegungswahrnehmung heraus. Erst wenn wir den Körper bewusst erfahren, haben wir eine Wahl. Dabei beruft sie sich u.a. auf Erfahrungen von Feldenkrais, E. Pickler und B. B. Cohen. In anderen Kapiteln erläutert sie, was in der medizinischen Auffassung von Geburt, in der Bewegungsentwick- lung, im Schulsystem und in der technisierten Gesellschaft „schief läuft“, warum es zu Lernstörungen kommt und welche Theorien von ADHS existieren.

Warum die frühe Berührung, die aufmerksame Hinwendung in der Begleitung und die eigene kinästhetische Kompetenz Grundvoraussetzungen für Angstfreiheit, Explorationstrieb und kognitives Lernen darstellen, wird u.a. begründet mit dem Aufbau des Gehirns nach Roth, mit D. Siegels interpersoneller Neurobiologie, mit Stresstheorien nach Hannaford, mit der grundlegenden Bedeutung des Spiels in der Entwicklungspsychologie und mit der Qualität der Eltern-Kind-Beziehung nach Winnicot.

Wer sich für die Wichtigkeit der bewegungsorientierten kinästhetischen Erziehung in der Bildungslandschaft engagiert, findet hier viele Begründungen und Erfahrungen sowie auch praktisches Übungsmaterial, das Eva Weißmann aus ihrem langjährigen Erfahrungsschatz zur Verfügung stellt.

In den Kapiteln über den Aufbau des Gehirns bleiben manche Begründungen noch vage, Zuordnungen sind noch konzeptionell. Da wünschte ich mir genauere Definitionen und einfachere Erklärungen des Körper-Geist-Zusammenhangs im Lernprozess. Doch insgesamt ist Weißmann ein sehr anregendes und auch Bewusstheit schaffendes Buch gelungen.

\section{Bettina Rollwagen}

DOI 10.2378/ktb2018.art19d 